the endocardial linear lesion with the roofline resulted in conversion to sinus rhythm (Figure $1, B$ and $C$ ).

Bilateral video-assisted thoracoscopic PV isolation is a safe, beating-heart approach for curative surgical treatment of AF. ${ }^{1}$ Linear lesions are known to improve the outcome of catheter ablation in patients with persistent atrial fibrillation. ${ }^{2}$ The left fibrous trigone line was introduced by Edgerton and colleagues. ${ }^{3}$ This linear lesion serves as an alternative to the endocardial mitral isthmus line extending from the left inferior PV to the mitral valve annulus. The creation of a completely transmural left fibrous trigone line can be hampered by the presence of epicardial fat. Not completely transmural lesions exhibit zones of low voltages and conduction slowing and can become pro-arrhythmic. ${ }^{4}$ In our patient, an incomplete left fibrous trigone line resulted in left atrial flutter. Most of these iatrogenic arrhythmias are very symptomatic. To prevent, as much as possible, such reentry circuits from occurring, it is of paramount importance to prove complete transmurality of each deployed linear lesion. This can effectively be done using an epicardial approach or a combined simultaneous thoracoscopic surgical and transvenous catheter procedure. ${ }^{5}$

\section{References}

1. Wolf RK, Schneeberger EW, Osterday R, Miller D, Merrill W, Flege JB, et al Video-assisted bilateral pulmonary vein isolation and left atrial appendage exclusion for atrial fibrillation. $J$ Thorac Cardiovasc Surg. 2005;130:797-802.

2. Willems S, Klemm H, Rostock T, Brandstrup B, Ventura R, Steven D, et al. Sub strate modification combined with pulmonary vein isolation improves outcome of catheter ablation in patients with persistent atrial fibrillation: a prospective randomized comparison. Eur Heart J. 2006;27:2871-8.

3. Edgerton JR, Jackman WM, Mack MJ. A new epicardial lesion set for minima access left atrial maze: the Dallas lesion set. Ann Thorac Surg. 2009;88:1655-7.

4. Sawhney N, Anousheh R, Chen W, Feld GK. Circumferential pulmonary vein ablation with additional linear ablation results in an increased incidence of left atrial flutter compared with segmental pulmonary vein isolation as an initial approach to ablation of paroxysmal atrial fibrillation. Circ Arrhythm Electrophysiol. 2010;3:243-8.

5. Pison L, La Meir M, Maessen J, Crijns HJ. Hybrid thoracoscopic surgical and transvenous catheter ablation of $\mathrm{AF}$, towards single procedure ablation of longstanding AF. Heart Rhythm. 2010;7(Suppl 5):S398.

\title{
Extremely rare but potential complication of diffuse brain edema due to air embolism during lung segmentectomy with selected segmental inflation technique by syringe needle during video-assisted thoracoscopic surgery
}

Tsunayuki Otsuka, MD, Yoshihiro Nakamura, MD, PhD, Aya Harada, MD, and Masami Sato, MD, PhD, Kagoshima, Japan

A 65-year-old man was diagnosed with right lung cancer in the upper lobe and S8 segment.

To avoid right pneumonectomy, he underwent right upper lobectomy and S8 segmentectomy by video-assisted thoracoscopic surgery. During segmentectomy, the A8 was ligated first, and then B8 was resected using an automatic stapler. An 18-gauge needle was inserted in the distal B8 bronchus without any blood regurgitation. After removing the inner needle, an air tube of a Bolheal Spray Set (The Chemo-Sero-Therapeutic Research Institute, Kumamoto, Japan) with a filter attached was connected to the outer

\footnotetext{
From the Department of General Thoracic Surgery, Kagoshima University Graduate School of Medical and Dental Sciences, Kagoshima, Japan.

Disclosures: Authors have nothing to disclose with regard to commercial support.

Received for publication July 20, 2011; accepted for publication July 27, 2011; available ahead of print Aug 29, 2011.

Address for reprints: Masami Sato, MD, PhD, Department of General Thoracic Surgery, Graduate School of Medical and Dental Sciences, Kagoshima University, 8-35-1 Sakuragaoka, Kagoshima, 890-8520 Japan (E-mail: m-sato@m2.kufm. kagoshima-u.ac.jp).

J Thorac Cardiovasc Surg 2011;142:e151-2

$0022-5223 / \$ 36.00$

Copyright (c) 2011 by The American Association for Thoracic Surgery doi:10.1016/j.jtcvs.2011.07.061
}

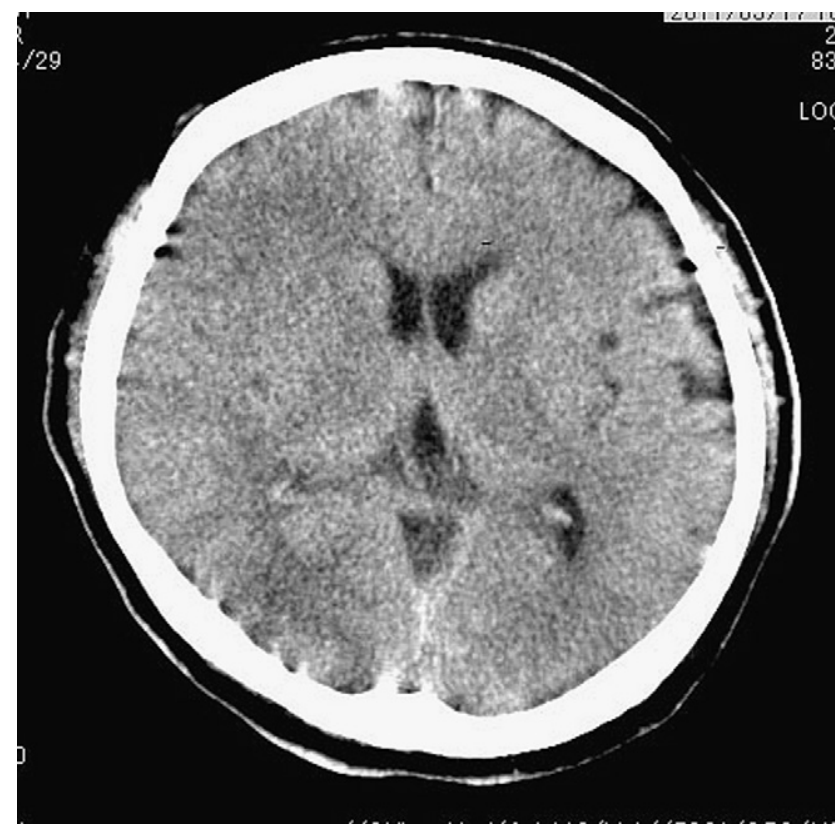

FIGURE 1. Computed tomography scan after hyperbaric oxygen therapy revealing diffuse brain edema. 


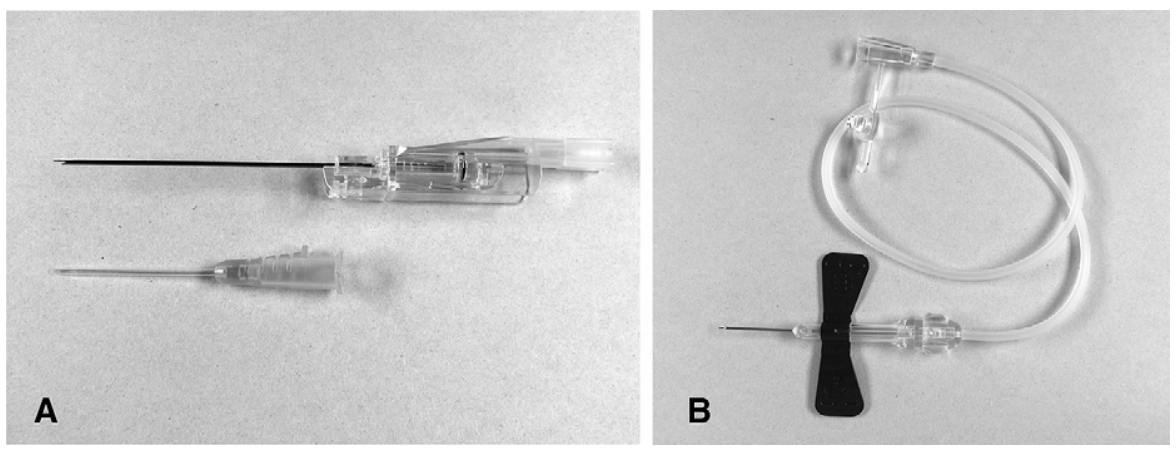

FIGURE 2. Needles used during lung segmentectomy with selected segmental inflation technique: A, inner needle (Upper) and outer sheath (Lower); $\mathrm{B}$, other type of needle (butterfly needle) used during lung segmentectomy with selected segmental inflation technique.

dwelling sheath, and air was blown into the tube. Because the air was not confirmed in the target area, which usually inflates, we stopped blowing air and removed the punctured in-dwelling needle. Immediately after this, a sudden elevation in blood pressure $(220 \mathrm{~mm} \mathrm{Hg} / 150 \mathrm{~mm} \mathrm{Hg})$ and a decline in end-tidal carbon dioxide pressure were observed, and ST-segment elevation on the electrocardiogram followed. On the transthoracic echocardiogram, air in the right atrium was observed and was diagnosed as an air embolism. Because the bronchus and pulmonary artery of the right S8 were already resected at this point, we decided to continue with the segmentectomy. The bronchial lumen was confirmed, and the sheath without the needle was inserted to blow air into the lumen. Segmentectomy using the automatic stapler was then completed. Immediately after the operation, hyperbaric oxygen therapy was performed. However, computed tomography images showed diffuse brain edema, and cerebral infarction was diagnosed (Figure 1). After 1 week of hypothermic therapy at $35^{\circ} \mathrm{C}$, the body temperature was restored, and the respirator was removed 2 weeks after the operation. Although symptoms of left hemiplegia and left spatial neglect were observed immediately after the first hyperbaric oxygen therapy, they were almost completely absent by 4 weeks after the operation.

With progress and the wide use of computed tomography, the number of smaller lesions being detected has been increasing, ${ }^{1}$ and segmentectomy with video-assisted thoracoscopic surgery has been one of the common methods. Because of the poor working spaces with video-assisted thoracoscopic surgery, several procedures for detecting the demarcation lines have been reported. Among them, the selected segmental inflation technique is frequently used in Japan. ${ }^{2,3}$ Some thoracic surgeons use a needle for this method in the operation field after the segmental bronchus has been resected (Figure 2), ${ }^{2}$ and some anesthesiologists blow out the air through the targeted bronchus with a thin bronchoscope before the segmental bronchus is resected. ${ }^{3}$

In our department, we had been using the selected segmental inflation technique with needles to avoid bacterial contamination in the operative field and to reduce the anesthesiologist's burden. We had no problems with this method before the present case. However, although no blood reflux was confirmed, the tip of the needle might have moved during inflation at this time with air blown into the vessels, which resulted in ST-segment elevation and diffuse brain edema. After the present case, we started using an "open-cut" method, in which the segmental bronchus is resected and opened, followed by insertion of the outer sheath without the needle, with air then blown into the lumen.

Before this experience, we had never seen such a complication. From the published data, only 1 case with the same complication was reported in 2010 in Japan, ${ }^{4}$ which suggests it is extremely rare, but possible, when a needle is used in the selected segmental inflation technique. Thus, we recommend an "open-cut" selected segmental inflation technique without the use of a needle or the use of the selected segmental inflation technique through a thin bronchoscope.

\section{References}

1. The National Lung Screening Trial Research Team. Reduced lung-cancer mortality with low-dose computed tomographic screening. N Engl J Med. 2011;365: 395-409.

2. Kamiyoshihara M, Kakegawa S, Morishita Y. Convenient and improved method to distinguish the intersegmental plane in pulmonary segmentectomy using a butterfly needle. Ann Thorac Surg. 2007;83:1913-4.

3. Okada M, Mimura T, Ikegaki J, Katoh H, Itoh H, Tsubota N. A novel videoassisted anatomic segmentectomy technique: selective segmental inflation via bronchofiberoptic jet followed by cautery cutting. J Thorac Cardiovasc Surg. 2007;133:753-8.

4. Kiribayashi M, Nakasone M, Moriyama N, Mochida S, Yamasaki K, Minami Y, et al. Multiple cerebral infarction by air embolism associated with remarkable low BIS value during lung segmentectomy with video assisted thoracic surgery (VATS) technique: a case report. Masui. 2010;59:480-3. 